The Astrophysical Journal, 662:730-737, 2007 June 10

(C) 2007. The American Astronomical Society. All rights reserved. Printed in U.S.A.

\title{
THE LINEAR SENSITIVITY OF HELIOSEISMIC RING DIAGRAMS TO LOCAL FLOWS
}

\author{
A. C. BIRCH \\ Colorado Research Associates, Northwest Research Associates, Inc., Boulder CO 80301; aaronb@cora.nwra.com \\ L. GIZON \\ Max-Planck-Institut für Sonnensystemforschung, Max-Planck-Strasse 2, 37191 Katlenburg-Lindau, Germany \\ AND \\ B. W. Hindman and D. A. Haber \\ JILA, University of Colorado, Boulder, CO 80309-440 \\ Received 2006 November 15; accepted 2007 February 7
}

\begin{abstract}
Ring-diagram analysis is a technique of local helioseismology used to infer plasma flows in the solar convection zone which generates intermediate data products known as ring-fitting parameters. Knowing the sensitivity of ringfitting parameters to actual flows in the Sun is important for interpreting these measurements. Working in plane-parallel geometry, we compute the linear sensitivity of ring-fitting parameters to small changes in the local power spectrum and then compute the sensitivity of the power spectrum to time-independent weak local flows. We combine these two results to obtain the three-dimensional Frechet kernels that give the linear sensitivity of ring-fitting parameters to both vertical and horizontal local mass flows. We find that ring measurements are essentially only sensitive to flows that are within the spatial region for which the ring diagram is computed. In addition, we find that the depth dependence of the sensitivity is essentially given by the mode kinetic energy density, as has traditionally been assumed. We show that the exact form of the sensitivity of ring measurements depends on the details of the fitting procedure.
\end{abstract}

Subject headings: scattering — Sun: helioseismology — Sun: oscillations — Sun: rotation — waves

\section{INTRODUCTION}

Ring-diagram analysis (Hill 1988) probes the spatial variation of solar flows and sound speed within the upper convection zone through measurement of the dispersion relation of high-degree acoustic waves traveling in this region. Advection of acoustic wave fronts by subsurface flows results in anisotropic shifts in the wave frequencies. More explicitly, waves traveling in opposite directions have their frequencies split relative to each other by the Doppler effect. Each frequency splitting, $\delta \omega$, can be seen as a measurement of some spatial average of the flow, with $\delta \omega=\boldsymbol{k} \cdot \boldsymbol{u}$, where $\boldsymbol{k}$ is the horizontal wavenumber and $\boldsymbol{u}$ is the measured average flow. Throughout this paper we refer to $\boldsymbol{u}=\left(u_{x}, u_{y}\right)$ as the ringfitting parameters, where $x$ and $y$ are the two horizontal coordinates. We obtain the linear sensitivity of ring-fitting parameters to spatially varying three-dimensional flows, $\boldsymbol{v}(\boldsymbol{r})=\left(v_{x}(\boldsymbol{r}), v_{y}(\boldsymbol{r}), v_{z}(\boldsymbol{r})\right)$, where $\boldsymbol{r}=(x, y, z)$ gives position in the solar interior. We will work in plane-parallel geometry, which is appropriate for ring diagrams computed over patches of the Sun that are small compared to the solar radius.

Traditionally, ring-diagram analysis has employed onedimensional inversions in depth to estimate subsurface flows from frequency splittings, using the assumption that the depth sensitivity of a frequency splitting is given by the kinetic energy density of the associated mode (e.g., Hill 1990; Patron et al. 1995; Basu et al. 1999; Haber et al. 2000, 2002; González Hernández et al. 2000; Komm et al. 2004). These estimated subsurface flows have been interpreted as representing horizontal spatial averages of the true flow field (e.g., Hindman et al. 2004).

Hindman et al. (2005) studied the horizontal sensitivity of ring diagrams to flows using a toy problem in two dimensions and concluded that the horizontal sensitivity was approximately the square of the apodization function (the function which selects the patch over which to compute the local power spectrum).
The generalization of this conclusion to three dimensions is not clear.

Here, we will show that the shape of the horizontal sensitivity functions depends on the range in frequency space around the resonance which is used in the ring fitting. We will also show that the assumption that the depth dependence is given by the kinetic energy density of the associated mode is reasonable.

The derivation of sensitivity kernels for ring-diagram analysis presented here is quite similar to the derivations of kernels for time-distance helioseismology (Duvall et al. 1993) by Gizon \& Birch (2002) and Birch et al. (2004). In particular, we use the Born approximation to estimate the sensitivity of the wavefield to small changes in the background solar model (e.g., the introduction of weak local flows or changes in sound speed). In addition, in this paper, as in Gizon \& Birch (2002), the measurement procedure is simplified to provide a straightforward relationship between the perturbation to the wavefield and the perturbation to the measured helioseismic parameters (in the case presented here, the ring-fitting parameters).

This paper is organized as follows. In $\S 2$ we describe the process of obtaining local power spectra. In $\S 3$ we estimate the linear sensitivity of ring parameters to small changes in the local power spectrum. In $\S 4$ we use the first Born approximation to determine the linear sensitivity of the local power spectrum to spatially varying flows and obtain sensitivity kernels for ring-analysis measurements. A few examples of sensitivity kernels are given in $\S 5$. We conclude, in $\S 6$, with a brief discussion of the possible applications of these results.

\section{LOCAL POWER SPECTRA}

In local helioseismology the geometry is approximated as plane parallel. This approximation is typically valid over a small fraction of the solar disk for horizontal wavelengths that are much 
smaller than the solar radius. We denote the oscillation signal, e.g., the line-of-sight component of the velocity, by $\phi(x, t)$, where $\boldsymbol{x}=$ $(x, y)$ is a horizontal position vector on the solar surface (defined by height $z_{\mathrm{obs}}$ ) and $t$ is time. It is customary to consider the data cube $\phi(x, t)$ in a reference frame which is corotating with the Sun to remove the main component of rotation.

In ring-diagram analysis, the signal is spatially apodized by a function $A$ that depends on the distance from a target location $\boldsymbol{x}_{0}$. We denote by $\Psi$ the data cube after apodization,

$$
\Psi\left(\boldsymbol{x}, t ; \boldsymbol{x}_{0}\right)=A\left(\left\|\boldsymbol{x}-\boldsymbol{x}_{0}\right\|\right) \phi(\boldsymbol{x}, t) .
$$

For the remainder of this paper, we will choose our coordinate system such that the origin of the horizontal coordinates is at the center of the apodized region, i.e., $\boldsymbol{x}_{0}=\mathbf{0}$. For this paper, we will employ the apodization function of Haber et al. (2002):

$$
A(x)= \begin{cases}1, & x<a, \\ {\left[1-(x-a)^{2} /(b-a)^{2}\right]^{2},} & a \leq x \leq b, \\ 0, & x>b,\end{cases}
$$

with $a=0.469 L$ and $b=0.5 L$, where $L$ is the diameter of the apodization region. In many applications, $L$ is of order $180 \mathrm{Mm}$, although measurements have been made with much smaller values of $L$ (e.g., Hindman et al. 2004).

The apodized signal, $\Psi$, is then Fourier transformed to give the local power spectrum, $P$,

$$
P(\boldsymbol{k}, \omega)=\left|h_{k}^{2} \sum_{\boldsymbol{k}^{\prime}} A\left(\boldsymbol{k}-\boldsymbol{k}^{\prime}\right) \phi\left(\boldsymbol{k}^{\prime}, \omega\right)\right|^{2},
$$

where $\boldsymbol{k}$ is the horizontal wavevector, $\omega$ is the temporal frequency, and $h_{k}=2 \pi / L$ is the sampling in each component of the wavevector. We employ the conventions for discrete Fourier transforms from Gizon \& Birch (2004); in particular, we use $f(x, t)=$ $h_{k}^{2} h_{\omega} \sum_{\boldsymbol{k}, \omega} f(\boldsymbol{k}, \omega) e^{i \boldsymbol{k} \cdot \boldsymbol{x}-\omega t}$, where $h_{\omega}$ is the sampling in frequency space. Throughout this paper we will use the same symbol for a function and its Fourier transform and distinguish between the two cases by the argument, e.g., $f(\boldsymbol{k})$ is the two-dimensional spatial Fourier transform of $f(\boldsymbol{x})$.

\section{LINEARIZATION OF MAXIMUM LIKELIHOOD FITTING}

Flow estimates are obtained by fitting local power spectra, $P$, with models (e.g., Patron et al. 1995; Basu et al. 1999; Haber et al. 2002). Although different techniques have been used, here we follow the general approach of Bogart et al. (1995) and Schou \& Bogart (1998). In this approach, cylindrical cuts at constant wavenumber are extracted from the full three-dimensional power spectrum. The power spectrum in each cut at fixed $k=\|\boldsymbol{k}\|$ is a function of temporal frequency $\omega$ and the direction of $\boldsymbol{k}$ denoted by the angle $\theta$ measured counterclockwise from the $\hat{\boldsymbol{x}}$-direction. A uniform horizontal flow $\boldsymbol{u}$ would Doppler shift the power spectrum by an amount $\delta \omega=\boldsymbol{k} \cdot \boldsymbol{u}$. A more general internal flow, however, will cause a Doppler shift which depends on $k$ and on the radial order, $n$, of the modes of oscillations.

In the Schou \& Bogart (1998) approach, ring-diagram analysis consists of extracting, in $\omega-\theta$ space, a vector flow parameter $\boldsymbol{u}^{(k n)}$ for each value of $k$ and $n$. In general, the fitting of slices through the power spectrum at constant $k$ is done using a sixparameter fit; the fit simultaneously estimates two flow parameters $u_{x}$ and $u_{y}$ in addition to the amplitude of the power spectrum, the central frequency, the line width, and a background term (e.g., Haber et al. 2002). Inversion of the parameters $\boldsymbol{u}^{(k n)}$ is then used to estimate the physical flow field in the upper convection zone (e.g., Basu et al. 1998).

In this paper we will consider a simpler two-parameter fit, where only the flow parameters $\boldsymbol{u}^{(k n)}=\left(u_{x}^{(k n)}, u_{y}^{(k n)}\right)$ are treated as free. These two parameters extracted from the data should not be confused with the two horizontal components of the physical flow field inside the Sun (this distinction will become obvious in $\S 4)$. This fitting method is motivated, in part, by the need to facilitate the derivation of three-dimensional sensitivity kernels for flows. In $\omega-\theta$ space, bins of size $h_{\omega} h_{k} / k$ are nearly statistically independent. Therefore, the power spectrum follows an exponential probability density function. The joint probability density function (pdf) of the power at fixed wavenumber $k$ for the ridge with radial order $n$ is thus

$$
\operatorname{pdf}(P)=\prod_{\omega, \theta} \frac{1}{\bar{P}(\boldsymbol{k}, \omega)} \exp \left[-\frac{P(\boldsymbol{k}, \omega)}{\bar{P}(\boldsymbol{k}, \omega)}\right]
$$

where the product is over frequencies $\omega$ in a range around the resonant frequency of the mode $(k, n)$, and also over all angles $\theta$. The function $\bar{P}$ is the expectation value of $P$. Recall that at fixed $k$, the vector $\boldsymbol{k}=\hat{\boldsymbol{x}} k \cos \theta+\hat{\boldsymbol{y}} k \sin \theta$, which appears in equation (4), is only a function of $\theta$.

Let us denote by $P_{0}(k, \omega)$ a known reference power spectrum which does not depend on the direction of $\boldsymbol{k}$ and which is a good approximation to the expectation value of the observed local power spectrum. This reference power spectrum can be obtained, for example, from the model calculations of Birch et al. (2004). The model that we choose to fit to the data is

$$
\bar{P}(\boldsymbol{k}, \omega)=P_{0}(k, \omega)-\boldsymbol{k} \cdot \boldsymbol{u}^{(k n)} \partial_{\omega} P_{0}(k, \omega)+\ldots
$$

This model is motivated by the linearization of the Dopplershifted power spectrum and by the assumption that $P_{0}$ is a good representation of the expectation of the power spectrum in the absence of a flow.

The vector $\boldsymbol{u}^{(k n)}$ that provides the best fit to the data is obtained by maximizing the log likelihood, $\ln (\mathrm{pdf})$. As described in Appendix A, we obtain

$$
\boldsymbol{u}^{(k n)}=\sum_{\theta, \omega} \boldsymbol{W}^{(k n)}(\theta, \omega)\left[P(\boldsymbol{k}, \omega)-P_{0}(k, \omega)\right],
$$

where the two-dimensional vector $\boldsymbol{W}^{(k n)}$ is given by

$$
\mathbf{W}^{(k n)}(\theta, \omega)=-\frac{h_{\theta} \hat{\boldsymbol{k}}\left[P_{0}(k, \omega)\right]^{-2} \partial_{\omega} P_{0}(k, \omega)}{\pi k \sum_{\omega^{\prime}}\left[P_{0}\left(k, \omega^{\prime}\right)\right]^{-2}\left[\partial_{\omega^{\prime}} P_{0}\left(k, \omega^{\prime}\right)\right]^{2}},
$$

where $h_{\theta}$ is the grid spacing the $\theta$.

The aim of the above discussion was to provide some motivation for equations (6) and (7). From now on, however, we will use equation (6) as a definition for the ring parameters $\boldsymbol{u}^{(k n)}$. Later in this section we will show, empirically, that this definition for the ring parameters makes sense and is useful. Using a simple definition for the ring measurements will also prove to be very helpful for deriving the sensitivity of these measurements to internal solar flows (a similar approach was used by Gizon \& Birch [2002] in the case of time-distance helioseismology by introducing a simpler definition for travel time measurements). 


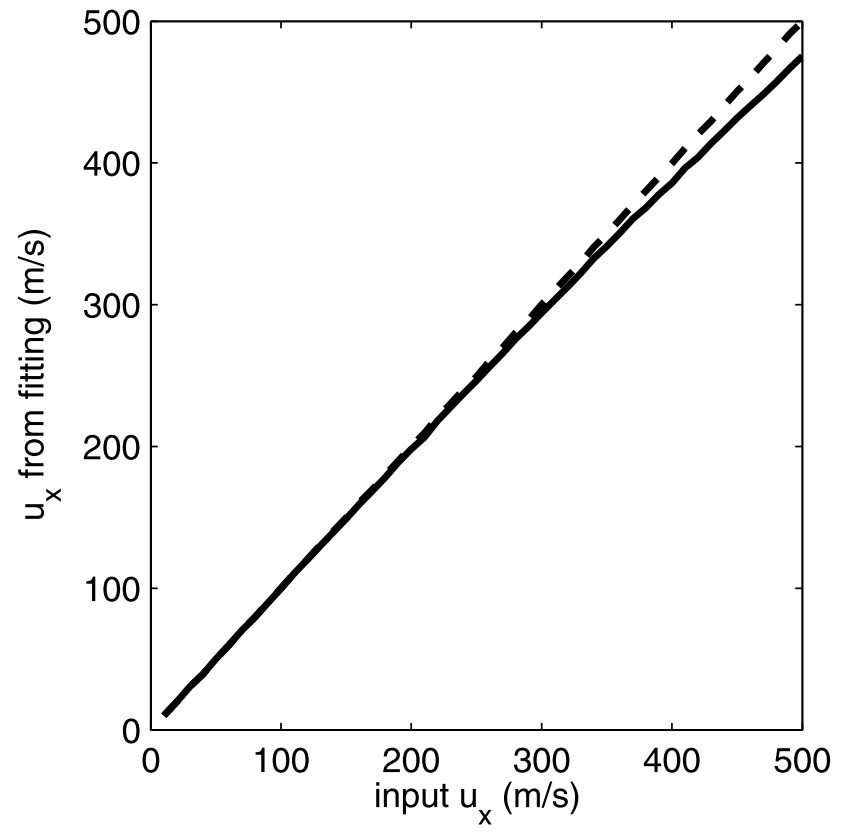

FIG. 1.- Results of nonlinear (dashed line) and linearized (eq. [6], solid line) fits to obtain $u_{x}$ from artificial power spectra. The horizontal axis is the known input $u_{x}$ in the model spectra. In both cases the vertical axis shows the mean $u_{x}$ resulting from the fit over 1000 realizations. The artificial spectra are realizations based on a Lorentizan limit spectrum with a line width and background similar to the $n=1, l=900$ mode as seen in MDI data. The linearization begins to deviate from the full nonlinear fit at about $200 \mathrm{~m} \mathrm{~s}^{-1}$. In general, we expect the linearization to be good when the frequency shift caused by the flow is small compared to the line width, so $u \lesssim \gamma / k$. For the case shown in this figure $\gamma / k \approx 200 \mathrm{~m} \mathrm{~s}^{-1}$, where $\gamma$ is the half-width at half-maximum of the power.

The linearization procedure used here could be generalized to treat other fitting methods. For example, in the case where smoothing of the observed power spectrum is performed in $\boldsymbol{k}$-space, the statistics of the resulting smooth power spectrum would no longer be exponential, the pdf given in equation (4) would not be appropriate, and the definition of the ring parameters given above would have to be modified.

The simple linearization expressed by equation (6) allows us to estimate the flow parameters $u_{x}$ and $u_{y}$ which result from changes in the observed power spectrum. In order for equation (6) to be useful, we must show that the linearization is valid. Figure 1 shows the results of a Monte Carlo simulation of both a nonlinear fitting procedure (maximizing eq. [4] numerically) and the linearization given in equation (6). In the simulation, artificial power spectra were generated by assuming a limit spectrum of the form $\bar{P}=$ $A\left[\left(\omega-\omega_{0}+u_{x} \cos \theta\right)^{2}+\gamma^{2}\right]^{-1}+b$ (where $A$ is the amplitude of the power spectrum, $\omega_{0}$ is the resonant frequency in the absence of flows, $\gamma$ is the damping rate, $u_{x}$ is the flow in the $\hat{\boldsymbol{x}}$-direction, and $b$ is a background) and exponential statistics. For each input value of $u_{x}$, we computed 1000 realizations of the power spectrum. In this way we can search for bias in the linearization. It appears that for flows less than about $u \lesssim \gamma / k$, the linearization is very similar to the full nonlinear fitting procedure. For $(n, k)$ values that are typically used in ring fitting, $\gamma / k$ is at least of order a few $100 \mathrm{~m} \mathrm{~s}^{-1}$. This suggests that computing sensitivity kernels based on the linearization given in equation (6) is sensible.

\section{RING-DIAGRAM KERNELS}

In this section we obtain the linear sensitivity of the power spectrum to local flows. By combining this result with the linearization of the dependence of the $\boldsymbol{u}^{(\mathrm{kn})}$ parameters on the power spectrum (eq. [6]), we will obtain the linear sensitivity of $\boldsymbol{u}^{(k n)}$ to a localized flow $\boldsymbol{v}(\boldsymbol{r})$. This linear sensitivity is encapsulated in kernel functions $K$ which satisfy

$$
u_{j}^{(k n)}=\sum_{s=x, y, z} \iiint_{\odot} d \boldsymbol{r} K_{j s}^{(k n)}(\boldsymbol{r}) v_{s}(\boldsymbol{r}) .
$$

The integration variable $\boldsymbol{r}$ ranges over the solar interior and summation is over the vector index $s=x, y, z$. There are two vectorvalued kernel functions, one for the $u_{x}(j=x)$ parameter and one for the $u_{y}(j=y)$ parameter. The kernel functions also depend on the radial order, $n$, and horizontal wavenumber, $k$, of the ring which is fit.

As discussed in $\S 2$, the power spectrum of the apodized wavefield is given by equation (3). The expected value of the power spectrum can be written as

$$
\begin{aligned}
P(\boldsymbol{k}, \omega)= & \iint_{-\infty}^{\infty} d \boldsymbol{k}_{1} \iint_{-\infty}^{\infty} d \boldsymbol{k}_{2} A\left(\boldsymbol{k}-\boldsymbol{k}_{1}\right) A^{*}\left(\boldsymbol{k}-\boldsymbol{k}_{2}\right) \\
& \times \mathrm{E}\left[\phi\left(\boldsymbol{k}_{1}, \omega\right) \phi^{*}\left(\boldsymbol{k}_{2}, \omega\right)\right]
\end{aligned}
$$

where for the sake of later convenience we have approximated the sums over all wavevectors as integrals. The symbol E denotes the expectation value, i.e., the average over many realizations of the stochastic wavefield $\phi$ (see e.g., Gizon \& Birch 2002 for a detailed discussion of ensemble averaging).

In order to compute the change in the power spectrum $P$ introduced by a local flow, $v(r)$, we need to compute the change in the underlying wavefield $\phi$ introduced by this flow. For the sake of simplicity we will consider only steady flows. There are many interesting solar flows, e.g., flows around active regions, which are approximately constant over the duration of roughly 1 day used to make typical ring-diagram measurements. The formalism developed here could in principle be extended to treat time-varying flows, in the manner of, e.g., Woodard (2006).

Following Gizon \& Birch (2002) and Birch et al. (2004), consider damped and driven solar oscillations with a displacement field $\boldsymbol{\xi}$ that obeys, to lowest order in the flow velocity $\boldsymbol{v}$,

$$
\left[\mathcal{L}_{0}+\delta \mathcal{L}\right] \boldsymbol{\xi}=\boldsymbol{S}
$$

with the wave equation operator in the absence offlows, $\mathcal{L}_{0}$, given by, e.g., Lynden-Bell \& Ostriker (1967),

$$
\begin{aligned}
\mathcal{L}_{0} \boldsymbol{\xi}= & \rho_{0} \ddot{\boldsymbol{\xi}}-\nabla\left[\gamma p_{0} \nabla \cdot \boldsymbol{\xi}+\boldsymbol{\xi} \cdot \nabla p_{0}\right] \\
& +(\nabla \cdot \boldsymbol{\xi}) \nabla p_{0}+\boldsymbol{\xi} \cdot \nabla\left(\nabla p_{0}\right)+\rho_{0} \partial_{t}(\Gamma \boldsymbol{\xi})
\end{aligned}
$$

where $\rho_{0}, p_{0}$, and $\gamma$ are the background density, pressure, and ratio of specific heats (we use the stratification from model S, Christensen-Dalsgaard et al. 1996, although truncated in depth). We have included a phenomenological damping operator, $\Gamma$ (see Birch et al. 2004). In addition we have used the Cowling approximation and neglected the variation of the gravitational acceleration with depth. The source function $S$ is intended to represent the driving of oscillations by near-surface turbulent convection. As shown in Gizon \& Birch (2002), in order to compute the expected power spectrum of the wavefield $\boldsymbol{\xi}$ we will only need the covariance of $\boldsymbol{S}$. As described below, we will use 

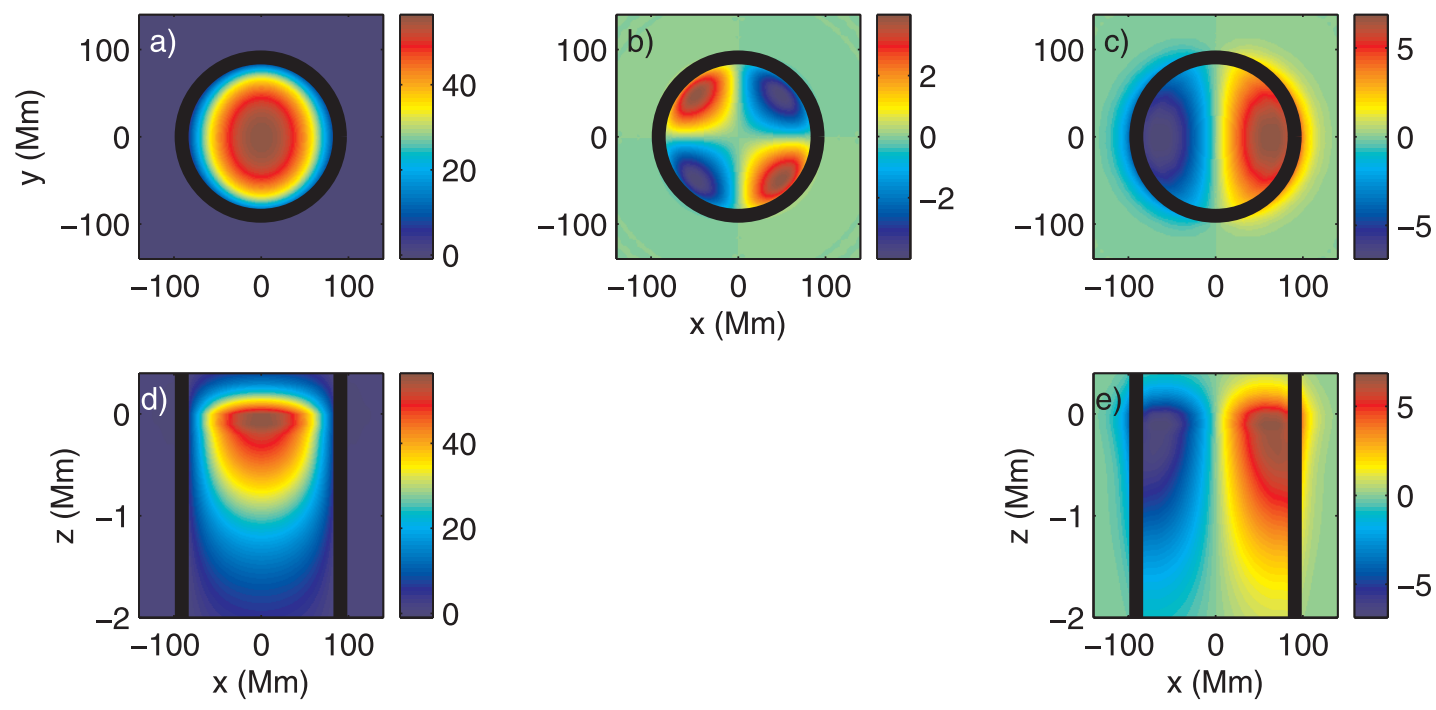

FIG. 2.- Sensitivity kernels for $u_{x}$ measurements for the case $n=0$ ( $f$-mode $)$ and $l=1092$. ( $a$ ) Horizontal slice, $200 \mathrm{~km}$ below the photosphere, through the sensitivity to a

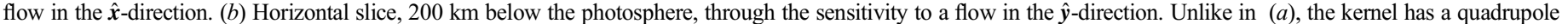

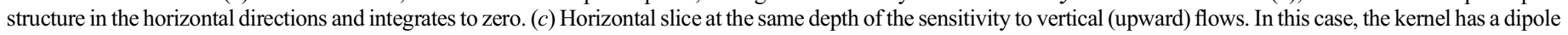

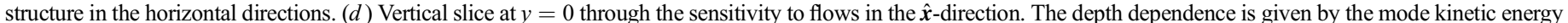

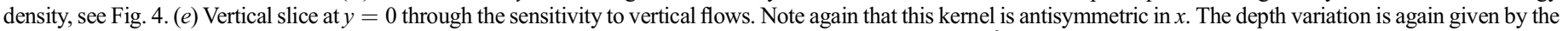
mode kinetic energy density. In all cases, the color-scale bars shows the value of the kernels in units of $10^{-6} \mathrm{Mm}^{-3}$.

the phenomenological model of Birch et al. (2004) for this covariance. The first-order perturbation to the wave equation introduced by a flow ${ }^{1}$ is

$$
\delta \mathcal{L} \boldsymbol{\xi}=2 \rho_{0} \partial_{t} \boldsymbol{v} \cdot \nabla \boldsymbol{\xi}
$$

Notice that this term only captures the direct advection of waves by the flow $\boldsymbol{v}$. Any associated changes in the background (nonwave) density and sound speed are neglected. We could in principle apply the Born approximation to model the linear effect of spatially inhomogeneous sound speed and density perturbations on the $\boldsymbol{u}^{(k n)}$ parameters. However, in this paper we consider only the direct effect of advection.

For the sake of simplicity, we assume that the observed wavefield, $\phi(\boldsymbol{x}, t)$, is the vertical component of the oscillation velocity. This assumption is reasonable for observations made near the center of the solar disk. Also for the sake of simplicity we assume that the observed wavefield is given by

$$
\phi(\boldsymbol{x}, t)=\partial_{t} \hat{\boldsymbol{z}} \cdot \boldsymbol{\xi}\left(\boldsymbol{x}, z_{\mathrm{obs}}, t\right),
$$

where $\hat{z}$ is an upward-directed unit vector and $z_{\text {obs }}$ is the height in the solar atmosphere where the observations are made. In this paper we choose the observation height $z_{\text {obs }}$ to be $200 \mathrm{~km}$ above the photosphere.

Before writing the power spectrum, let us briefly review some definitions and notation for Green's functions. Throughout this paper we employ the same Green's functions as Birch et al. (2004). The vector-valued causal Green's functions for a source in the $s$-direction at time $t=0$, horizontal position $\boldsymbol{x}=\mathbf{0}$, and source depth $z^{\prime}$ we denote as $\boldsymbol{G}^{S}\left(\boldsymbol{x}, t, z, z^{\prime}\right)$ and define as being the solution to

$$
\mathcal{L}^{0} \boldsymbol{G}^{S}\left(\boldsymbol{x}, t, z, z^{\prime}\right)=\hat{\boldsymbol{e}}_{s} \delta_{\mathrm{D}}(\boldsymbol{x}) \delta_{\mathrm{D}}(t) \delta_{\mathrm{D}}\left(z-z^{\prime}\right),
$$

\footnotetext{
1 See, e.g., lecture notes of J. Christensen-Dalsgaard on solar oscillations from 1994 at Institut for Fysik og Astronomi, Aarhus Universitet, at http://astro.phys.au.dk/ $\sim$ jcd/oscilnotes/.
}

where $\hat{\boldsymbol{e}}_{s}$ with $s=x, y, z$ are unit vectors along the coordinate axis and $\delta_{\mathrm{D}}$ denotes the Dirac delta function. We use the same boundary conditions as in Birch et al. (2004): zero Lagrangian pressure at the top of model S and no vertical motion at a depth of $300 \mathrm{Mm}$ below the photosphere (this bottom boundary condition has essentially no effect on the computations presented here). The vector-valued Green's function $\boldsymbol{G}^{s}\left(\boldsymbol{x}, t, z, z^{\prime}\right)$ gives the displacement response as a function of horizontal position $\boldsymbol{x}$, time $t$, and height $z$, to a delta function source in the $\hat{\boldsymbol{e}}_{s}$-direction at horizontal position $\mathbf{0}$, time $t=0$, and height $z^{\prime}$.

We also define the Green's functions for the wave sources of Birch et al. (2004)

$$
\boldsymbol{H}(\boldsymbol{x}, t, z)=\left.\partial_{z^{\prime}} \boldsymbol{G}^{z}\left(\boldsymbol{x}, t, z, z^{\prime}\right)\right|_{z^{\prime}=z_{\mathrm{src}}},
$$

where the source depth $z_{\text {src }}$ is chosen to be $100 \mathrm{~km}$ below the photosphere.

By combining equation (13), the Green's functions described above, the source model of Birch et al. (2004), and equation (9), we obtain a model for the ring power in the absence of flows

$$
\begin{aligned}
P_{0}(\boldsymbol{k}, \omega)= & (2 \pi)^{6} m(\omega) \iint_{-\infty}^{\infty} d \boldsymbol{k}^{\prime}\left|A\left(\boldsymbol{k}-\boldsymbol{k}^{\prime}\right)\right|^{2} \\
& \times\left|H_{z}\left(\boldsymbol{k}^{\prime}, \omega, z_{\mathrm{obs}}\right)\right|^{2} .
\end{aligned}
$$

In equation (16), $P_{0}$ is the model power spectrum in the absence of flows and $m(\omega)$ is the temporal source covariance given by Birch et al. (2004). The Green's function $H_{z}\left(\boldsymbol{k}^{\prime}, \omega, z_{\mathrm{obs}}\right)$ is the $z$-component of the Green's function defined in equation (15) at wavevector $\boldsymbol{k}^{\prime}$, temporal frequency $\omega$, and observation height $z_{\text {obs }}$.

Applying the Born approximation to equation (10) and following the same procedure that we used to obtain equation (16), 

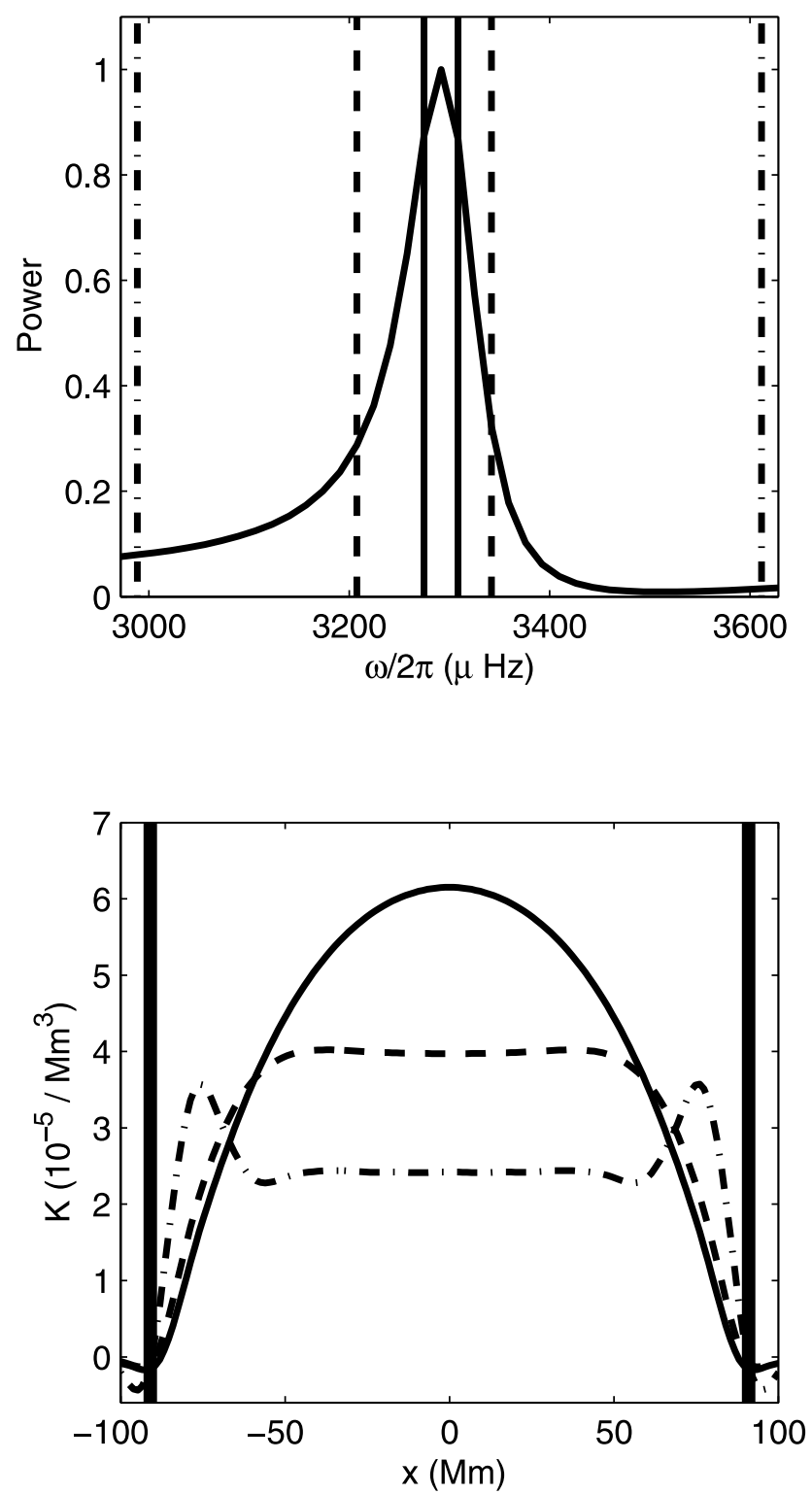

FIG. 3.-Examples of the dependence of the shape of the kernels on the width of the window in frequency used to fit the power spectrum. The top panel shows the unperturbed power spectrum of the $n=0$ ( $f$-mode), $l=1092$ resonance along with three different fitting intervals, shown as the vertical solid, dashed, and dot-dashed lines. The bottom panel shows slices through the corresponding $K_{x x}$ at $y=0$ and $z=0 \mathrm{Mm}$, the line styles correspond to the fitting intervals shown in the top panel. In the lower panel, the solid vertical lines show the edges of the spatial apodization region. As the fitting interval in frequency space is increased, the kernels become less localized around the center of the apodization region. The widest fitting interval (dot-dashed line) covers $40 \%$ of the distance (in frequency) between $f$ and $p_{1}$ and is the fitting interval used in Haber et al. (2002).

we obtain the first-order perturbation to power spectrum caused by a local flow $\boldsymbol{v}(\boldsymbol{r})$,

$$
\delta P(\boldsymbol{k}, \omega)=\iiint_{\odot} d \boldsymbol{r} \boldsymbol{C}(\boldsymbol{k}, \omega ; \boldsymbol{r}) \cdot \boldsymbol{v}(\boldsymbol{r}),
$$

with the sensitivity function $\mathbf{C}$ given by

$$
C_{s}(\boldsymbol{k}, \omega ; \boldsymbol{r})=2 m(\omega) \operatorname{Re}\left[\boldsymbol{I}^{s}(\boldsymbol{k}, \omega ; \boldsymbol{r}) \cdot \boldsymbol{I I}(\boldsymbol{k}, \omega ; \boldsymbol{r})\right] .
$$

The symbol Re denotes the real part. The integrals $\boldsymbol{I}^{s}(s=x, y, z)$ and $\boldsymbol{I I}$ are vector-valued inverse Fourier transforms, in two spa-

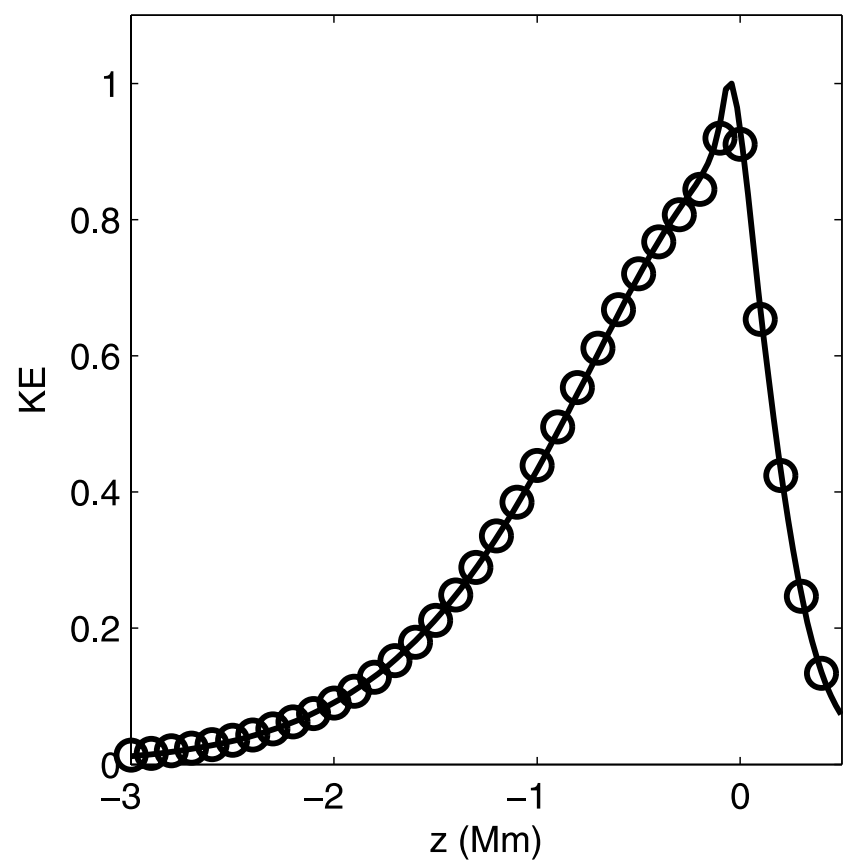

FIG. 4.-Kinetic energy density (solid line) and horizontal integral of the kernel (circles) as functions of depth. This example is for the kernel shown in Fig. 2. Both functions have been normalized.

tial dimensions, of products of Green's functions and Fourier transforms of the window function, and are given explicitly in Appendix B. The function $m(\omega)$ is the temporal part of the source covariance and is described in detail by Birch et al. (2004).

Combining equations (6) and (17) with the definition of the ring kernels (eq. [8]) we have

$$
K_{j s}^{(k n)}(\boldsymbol{r})=\sum_{\theta, \omega} W_{j}^{(k n)}(\theta, \omega) C_{s}(\boldsymbol{k}, \omega ; \boldsymbol{r}),
$$

where the sum over is taken over all pixels $(\theta, \omega)$ in the unwrapped power spectrum. The range in $\omega$ around the resonance we leave as arbitrary for now; this issue will be discussed in detail in $\S 5$. The function $C$, the linear sensitivity of the power spectrum to flows, is defined in equation (18), and the function $\boldsymbol{W}$, the linear sensitivity of the fit parameters $\boldsymbol{u}^{(k n)}$ to changes in the power spectrum, is defined in equation (7). As described in equation (8), the function $K_{j s}^{(k n)}(\boldsymbol{r})$ gives the linear sensitivity of the fit parameter $u_{j}^{(k n)}$, $j=x, y$, to a flow in the direction $s=x, y, z$ at the location $\boldsymbol{r}$ in the solar interior.

\section{EXAMPLE CALCULATIONS}

Figure 2 shows example kernels $K$ for the sensitivity of the $u_{x}$ parameter to flows in the $\hat{\boldsymbol{x}}$-, $\hat{\boldsymbol{y}}$-, and $\hat{\boldsymbol{z}}$-directions, for the case where the radial order and angular degree are, respectively, $n=0$ and $l=1092$. The range in $\omega$ that was used in the computation of the kernel (eq. [19]) is shown by the solid lines in the top panel of Figure 3 . The apodization function $A$ is given by equation (2) with $L=182 \mathrm{Mm}$ (about 15 heliocentric degrees). The kernel for the flow in the $\hat{\boldsymbol{x}}$-direction, $K_{x x}$, is azimuthally symmetric around the origin and is zero outside of the apodization region. The kernel for the flow in the $\hat{\boldsymbol{y}}$-direction, $K_{x y}$ has a quadrupole symmetry and integrates to zero. A uniform flow in the $\hat{\boldsymbol{y}}$-direction does not affect the $u_{x}$ parameter to first order. However, localized flows in the $\hat{\boldsymbol{y}}$-direction generate scattered waves. These scattered waves alter the local ring power spectrum, and as a result can influence the $u_{x}$ 

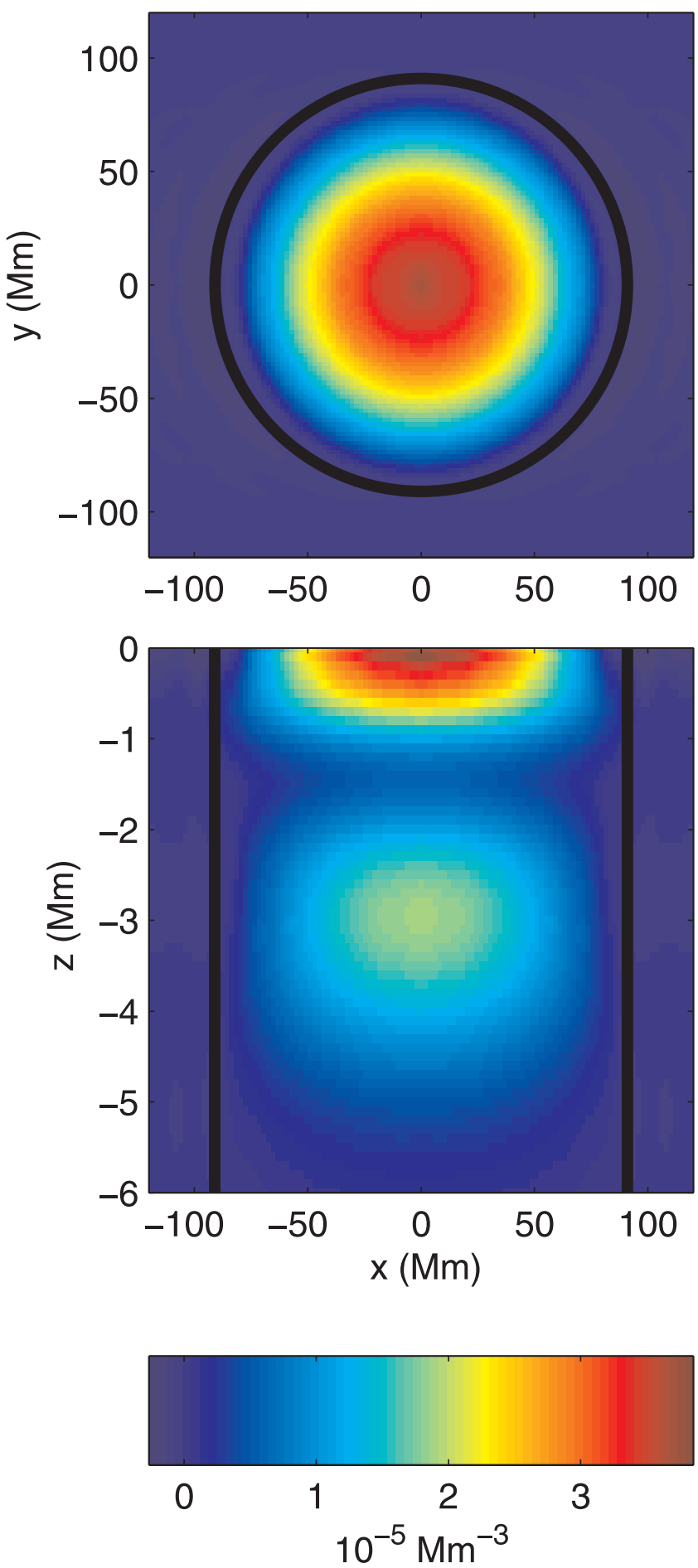

FIg. 5.-An example kernel for the sensitivity of the $u_{x}$ parameter to a flow in the $\hat{x}$-direction for the case $n=1$ and $l=716$. The top panel shows a horizontal slice at $z=-200 \mathrm{~km}$. The black circle outlines the outer edge of the apodization region (eq. [2], with $L=182 \mathrm{Mm}$ ). The lower panel shows a vertical slice in the plane $y=0$

measurement. Notice that by symmetry, the kernel $K_{x y}$ is zero in the planes $x=0$ and $y=0$. In the same manner, a localized flow in the $\hat{z}$-direction in general produces a scattered wave, and so the sensitivity of $u_{x}$ to local vertical flows, $K_{x z}$, is not zero, although the total integral of this kernel is zero. A local vertical flow at $x=0$ cannot distinguish between the $\hat{\boldsymbol{x}}$ - and $-\hat{\boldsymbol{x}}$-direction. As a result, $K_{x z}$ is zero in the plane $x=0$.
As shown in Figure 3, the shape of the kernels in the horizontal directions in general depends on the width in frequency space of the neighborhood of the resonance that is used to do the fitting, i.e., the range in $\omega$ that is employed in equation (19). This is possible, as the ring power spectrum at different values of $\omega$ has different spatial sensitivity functions $\boldsymbol{C}(\boldsymbol{k}, \omega ; \boldsymbol{r})$. Near resonance, the functions $\boldsymbol{C}$ are smooth and localized around the center of the apodization region. Off resonance, the function $\boldsymbol{C}$ can be complicated.

Traditionally, it has been assumed that ring measurements have a sensitivity to flows that varies with depth as the mode kinetic energy density. The horizontal average of the kernel is well approximated by the kinetic energy density $\rho_{0}\left\|\boldsymbol{\xi}_{n}(k ; z)\right\|^{2}$, where $\boldsymbol{\xi}_{n}(k ; z)$ is the eigenfunction of the mode with radial order $n$ and horizontal wavenumber $k$ (Fig. 4). Figure 4 suggests that the traditional approximation of using global-mode rotation kernels to invert ringdiagram measurements is reasonable.

Figure 5 shows an example kernel $K_{x x}$ for the case $n=1$ and $l=716$. As with the previous example, the kernel $K_{x x}$ is nearly azimuthally symmetric, with the sensitivity somewhat concentrated near the center of the apodization region. The depth dependence shows the basic structure of kinetic energy density of the $n=1$ mode.

The simple plane-parallel background (unperturbed) model presented here is horizontally homogeneous and isotropic. As a result, the kernels for the $u_{y}$ parameter can be obtained by a change of coordinates from the kernels for the $u_{x}$ parameter. In particular,

$$
\begin{aligned}
& K_{y x}(x, y, z)=K_{x y}(y, x, z), \\
& K_{y y}(x, y, z)=K_{x x}(y, x, z), \\
& K_{y z}(x, y, z)=K_{x z}(y, x, z) .
\end{aligned}
$$

These kernels can be visualized from the $K_{x}$ kernels as reflections over the line $x=y$.

\section{DISCUSSION}

We have derived, in the Born approximation, the threedimensional sensitivity of ring-diagram measurements to local flows. The sensitivity of $u_{x}$ to flows in the $\hat{\boldsymbol{x}}$-direction $\left(K_{x x}\right)$ is nearly zero outside of the apodization region and has a depth dependence given by the mode kinetic energy density. The sensitivity to flows in the $\hat{\boldsymbol{y}}(\hat{z})$ direction shows quadrupole (dipole) structure in the horizontal directions and integrates to zero.

The sensitivity functions described here are expected to reasonably represent the effects of weak local flows on ring measurements, and as a result may be incorporated into inversions of ring-diagram measurements. We emphasize that $u_{x}$ measurements are not only sensitive to flows in the $\hat{\boldsymbol{x}}$-directions. In particular, spatially nonuniform flows in the other directions can alter $u_{x}$. For large-scale flows, however, these effects are small. For example, a rough estimate for a giant cell-like flow, with $m=8$ and a nearsurface vertical velocity that is an order of magnitude smaller than the horizontal velocity, suggests contributions from the $\hat{\boldsymbol{y}}$ - and $\hat{z}$-components of the flow that are about $1 \%$ of the contribution from the $\hat{\boldsymbol{x}}$-component. Estimates are difficult for smaller scale flows, where the vertical structure of the flow is also important. Inversions may be useful in untangling these effects.

In this paper we have only computed the direct effect of advection by local flows; we expect that changes in sound speed and density may in general accompany these flows. Generalizing from our results here, we expect that horizontally inhomogeneous sound speed and density variations will also perturb the $\boldsymbol{u}$ measurements. In addition, in this paper we addressed only the 
question of time-independent mass flows. The extension to nonsteady flows is possible within the framework presented here. In addition, extension to spherical geometry would also be relatively straightforward.

Ring-diagram analysis was not originally designed for probing flows at the small spatial scales. In particular, we emphasize that the derivation of kernels for the effects of vertical flows on the $\boldsymbol{u}$ parameters does not imply that vertical flows can be inferred from the $\boldsymbol{u}$ parameters with a reasonable ratio of signal to noise. It may be that time-distance helioseismology (Duvall et al. 1993) or helioseismic holography (Lindsey \& Braun 1997) are better suited to direct measurement of vertical flows.

The work of A. C. B. was supported by NASA contract NNH04CC05C. B. H. and D. H. acknowledge support from NASA NAG5-12491, NSFATM-0219581, NASA NNG05GM83G, and NASA NAG5-13520.

\section{APPENDIX A}

\section{LINEARIZATION OF MAXIMUM LIKELIHOOD FITTING}

In this Appendix we derive equations (6) and (7). We begin by taking the logarithm of equation (4) to obtain

$$
\ln (\text { pdf })=-\sum_{\theta, \omega}\left[\frac{P(\boldsymbol{k}, \omega)}{\bar{P}(\boldsymbol{k}, \omega)}+\log \bar{P}(\boldsymbol{k}, \omega)\right]
$$

where $\theta$ gives the angle between $\boldsymbol{k}$ and the $x$-axis, and $\omega$ is the temporal frequency. The sum over $\omega$ is taken over the range of frequency that is used in the maximum likelihood fitting and $\theta$ ranges from 0 to $2 \pi$. The model power spectrum $\bar{P}$ is a function of the velocity parameters $\boldsymbol{u}^{(k n)}$. Throughout this Appendix we will use the short notation $\boldsymbol{u}=\boldsymbol{u}^{(\mathrm{kn})}$. Maximization of equation (A1) with respect to $\boldsymbol{u}$ requires

$$
0=\nabla_{\boldsymbol{u}} \ln (\mathrm{pdf})=\sum_{\theta, \omega} \frac{P(\boldsymbol{k}, \omega)-\bar{P}(\boldsymbol{k}, \omega)}{\bar{P}^{2}(\boldsymbol{k}, \omega)} \nabla_{\boldsymbol{u}} \bar{P}(\boldsymbol{k}, \omega)
$$

where $\nabla_{\boldsymbol{u}}$ represents the gradient with respect to the vector $\boldsymbol{u}$. Equation (A2) is a general result that does not assume any particular form of the model power spectrum $\bar{P}$.

We now assume that the model power spectrum is given by equation (5),

$$
\bar{P}(\boldsymbol{k}, \omega)=P_{0}(k, \omega)-\boldsymbol{k} \cdot \boldsymbol{u} \partial_{\omega} P_{0}(k, \omega)+\ldots
$$

We want to obtain an approximate solution to equation (A2) in the limit of small $\boldsymbol{u}$, in particular when the Doppler shift $\delta \omega=\boldsymbol{k} \cdot \boldsymbol{u}$ is small compared to the line width of the resonance that we are fitting. In this case we can take only the first two terms in the expansion (eq. [A3]) of the model power spectrum. Equation (A2) then becomes

$$
\sum_{\theta, \omega} \frac{P-P_{0}+\boldsymbol{k} \cdot \boldsymbol{u} \partial_{\omega} P_{0}}{\left[P_{0}-\boldsymbol{k} \cdot \boldsymbol{u} \partial_{\omega} P_{0}\right]^{2}} P_{0}=0
$$

Recall that the functions $P$ and $P_{0}$ are functions of horizontal wavenumber, $\boldsymbol{k}$, and frequency, $\omega$. We have simplified the notation for the sake of readability. With the assumption that $P-P_{0}$ is the same order as $\boldsymbol{u} \cdot \boldsymbol{k} \partial_{\omega} P_{0}$, the denominator can be approximated as $P_{0}^{2}$ without losing any leading terms, and we have

$$
h_{\theta} \sum_{\theta, \omega} P_{0}^{-2}\left(P-P_{0}+\boldsymbol{k} \cdot \boldsymbol{u} \partial_{\omega} P_{0}\right) \boldsymbol{k} \partial_{\omega} P_{0}=0
$$

For later convenience, we have multiplied the equation by the grid spacing in the $\theta$-direction, $h_{\theta}$.

Reorganizing equation (A5) gives

$$
h_{\theta} \sum_{\theta, \omega}(\boldsymbol{k} \cdot \boldsymbol{u}) \boldsymbol{k} P_{0}^{-2}\left(\partial_{\omega} P_{0}\right)^{2}=-h_{\theta} \sum_{\theta, \omega} \boldsymbol{k} P_{0}^{-2}\left(P-P_{0}\right) \partial_{\omega} P_{0} .
$$

Note that for $\boldsymbol{u}=u(\cos \phi, \sin \phi)$, we have

$$
\begin{aligned}
h_{\theta} \sum_{\theta}(\boldsymbol{k} \cdot \boldsymbol{u}) \boldsymbol{k} & =u k^{2} h_{\theta} \sum_{\theta} \cos (\theta-\phi)(\cos \theta, \sin \theta) \\
& =\pi k^{2} \boldsymbol{u} .
\end{aligned}
$$


We have approximated the sums $h_{\theta} \sum_{\theta} \cos ^{2} \theta=h_{\theta} \sum_{\theta} \sin ^{2} \theta$ as $\int_{0}^{2 \pi} \cos ^{2} \theta \mathrm{d} \theta=\pi$. This allows simplification of equation (A6) to

$$
\boldsymbol{u}=-\frac{h_{\theta} \sum_{\theta, \omega} \boldsymbol{k} P_{0}^{-2}\left(P-P_{0}\right) \partial_{\omega} P_{0}}{\pi k^{2} \sum_{\omega} P_{0}^{-2}\left(\partial_{\omega} P_{0}\right)^{2}} .
$$

Equations (6) and (7) can be obtained directly from this result.

\section{APPENDIX B \\ INTEGRALS}

In this Appendix we record the linear sensitivity of the power spectrum to local flows, described in $\S 4$. The linear sensitivity of the power spectrum to local mass flows is given by the function $\boldsymbol{C}$ which satisfies

$$
\delta P(\boldsymbol{k}, \omega)=\iiint_{\odot} d \boldsymbol{r} \boldsymbol{C}(\boldsymbol{k}, \omega ; \boldsymbol{r}) \cdot \boldsymbol{v}(\boldsymbol{r}) .
$$

The function $\boldsymbol{C}$ is given by

$$
C_{s}(\boldsymbol{k}, \omega ; \boldsymbol{r})=2 m(\omega) \operatorname{Re}\left[\boldsymbol{I}^{s}(\boldsymbol{k}, \omega ; \boldsymbol{r}) \cdot \boldsymbol{I I}(\boldsymbol{k}, \omega ; \boldsymbol{r})\right]
$$

with the integrals $\boldsymbol{I}$ and $\boldsymbol{I I}$ given by

$$
\begin{aligned}
& \boldsymbol{I}^{x}(\boldsymbol{k}, \omega ; \boldsymbol{r})=-2(2 \pi)^{4} \omega \rho_{0}(z) \iint_{-\infty}^{\infty} d \boldsymbol{k}^{\prime}\left(\hat{\boldsymbol{x}} \cdot \boldsymbol{k}^{\prime}\right) A^{*}\left(\boldsymbol{k}-\boldsymbol{k}^{\prime}\right) H_{z}^{*}\left(\boldsymbol{k}^{\prime}, \omega, z_{\mathrm{obs}}\right) \boldsymbol{H}\left(\boldsymbol{k}^{\prime}, \omega, z\right) e^{i \boldsymbol{k}^{\prime} \cdot \boldsymbol{x}}, \\
& \boldsymbol{I}^{y}(\boldsymbol{k}, \omega ; \boldsymbol{r})=-2(2 \pi)^{4} \omega \rho_{0}(z) \iint_{-\infty}^{\infty} d \boldsymbol{k}^{\prime}\left(\hat{\boldsymbol{y}} \cdot \boldsymbol{k}^{\prime}\right) A^{*}\left(\boldsymbol{k}-\boldsymbol{k}^{\prime}\right) H_{z}^{*}\left(\boldsymbol{k}^{\prime}, \omega, z_{\mathrm{obs}}\right) \boldsymbol{H}\left(\boldsymbol{k}^{\prime}, \omega, z\right) e^{i \boldsymbol{k}^{\prime} \cdot \boldsymbol{x}}, \\
& \boldsymbol{I}^{z}(\boldsymbol{k}, \omega ; \boldsymbol{r})=2 i(2 \pi)^{4} \omega \rho_{0}(z) \iint_{-\infty}^{\infty} d \boldsymbol{k}^{\prime} A^{*}\left(\boldsymbol{k}-\boldsymbol{k}^{\prime}\right) H_{z}^{*}\left(\boldsymbol{k}^{\prime}, \omega, z_{\mathrm{obs}}\right) \partial_{z} \boldsymbol{H}\left(\boldsymbol{k}^{\prime}, \omega, z\right) e^{i \boldsymbol{k}^{\prime} \cdot \boldsymbol{x}}, \\
& I I_{s}(\boldsymbol{k}, \omega ; \boldsymbol{r})=(2 \pi)^{3} \iint_{-\infty}^{\infty} d \boldsymbol{k}^{\prime} A\left(\boldsymbol{k}-\boldsymbol{k}^{\prime}\right) G_{z}^{s}\left(\boldsymbol{k}^{\prime}, \omega, z_{\mathrm{obs}}, z\right) e^{-i \boldsymbol{k}^{\prime} \cdot \boldsymbol{x}},
\end{aligned}
$$

where $\boldsymbol{r}=(\boldsymbol{x}, z)$ and $i=\sqrt{-1}$. The Green's functions $\boldsymbol{G}$ and $\boldsymbol{H}$ are defined in equations (14) and (15), respectively.

\section{REFERENCES}

Basu, S., Antia, H. M., \& Tripathy, S. C. 1998, in SOHO 6/GONG 98: Structure and Dynamics of the Interior of the Sun and Sun-like Stars (ESA SP-418; Noordwijk: ESA), 705 1999, ApJ, 512, 458

Birch, A. C., Kosovichev, A. G., \& Duvall, T. L. 2004, ApJ, 608, 580

Bogart, R. S., et al. 1995, in Proc. of the 4th SOHO Workshop: Helioseismology (ESA SP-376; Noordwijk: ESA), 147

Christensen-Dalsgaard, J., et al. 1996, Science, 272, 1286

Duvall, T. L., Jefferies, S. M., Harvey, J. W., \& Pomerantz, M. A. 1993, Nature, 362,430

Gizon, L., \& Birch, A. C. 2002, ApJ, 571, 966 2004, ApJ, 614, 472

González Hernández, I., Patrón, J., Roca Cortés, T., Bogart, R. S., Hill, F., \& Rhodes, Jr., E. J. 2000, ApJ, 535, 454

Haber, D. A., Hindman, B. W., Toomre, J., Bogart, R. S., Larsen, R. M., \& Hill, F. 2002, ApJ, 570, 855

Haber, D. A., Hindman, B. W., Toomre, J., Bogart, R. S., Thompson, M. J., \& Hill, F. 2000, Sol. Phys., 192, 335
Hill, F. 1988, ApJ, 333, 996

1990, Sol. Phys., 128, 321

Hindman, B. W., Featherstone, N. A., Haber, D. A., Musman, S., \& Toomre, J. 2004, in Proc. SOHO 14/GONG 2004 Workshop: Helio- and Asteroseismology (ESA SP-559; Noordwijk: ESA), 460

Hindman, B. W., Gough, D., Thompson, M. J., \& Toomre, J. 2005, ApJ, 621, 512

Komm, R., Corbard, T., Durney, B. R., González Hernández, I., Hill, F., Howe, R., \& Toner, C. 2004, ApJ, 605, 554

Lindsey, C., \& Braun, D. C. 1997, ApJ, 485, 895

Lynden-Bell, D., \& Ostriker, J. P. 1967, MNRAS, 136, 293

Patron, J., Hill, F., Rhodes, E. J., Korzennik, S. G., \& Cacciani, A. 1995, ApJ 455,746

Schou, J., \& Bogart, R. S. 1998, ApJ, 504, L131

Woodard, M. F. 2006, ApJ, 649, 1140 